\title{
Adolescents' ability to select healthy food using two different front-of-pack food labels: a cross-over study
}

\author{
Nancy Babio ${ }^{1}{ }^{2}$, Paloma Vicent ${ }^{1}$, Leonor López ${ }^{1}$, Anna Benito ${ }^{1}$, Julio Basulto ${ }^{3}$ and \\ Jordi Salas-Salvadó ${ }^{1,2, *}$ \\ ${ }^{1}$ Human Nutrition Unit, Faculty of Medicine and Health Sciences, Hospital Universitari de Sant Joan, \\ Departament de Bioquímica i Biotecnologia, IISPV, Universitat Rovira i Virgili, 43201 Reus, Spain: \\ ${ }^{2}$ Centro de Investigación Biomédica en Red Fisiopatologia de la Obesidad y Nutrición (CIBERobn), \\ Instituto de Salud Carlos III, Madrid, Spain: ${ }^{3}$ Review, Study and Scientific Position Statement Group of the \\ Spanish Association of Dietitians-Nutritionists (GREP-AEDN), Spain
}

Submitted 5 July 2012: Final revision received 20 March 2013: Accepted 5 April 2013: First published online 17 May 2013

\begin{abstract}
Objective: To compare, in adolescents, two models of front-of-pack Guideline Daily Amounts (GDA) labels in terms of (i) friendliness and acceptance and (ii) the ability to choose a diet that closely follows the nutritional recommendations.

Design: A randomized cross-over study was designed to compare two simplified front-of-pack GDA nutrition labels.

Setting: A Spanish secondary school.

Subjects: Eighty-one healthy adolescents aged between 14 and 16 years were recruited. Participants were randomly exposed to two experimental non-real food-choice conditions using multiple-traffic-light or monochrome nutritional labels. Participants had to choose options from a closed menu for $5 \mathrm{~d}$ on the basis of the experimental front-of-pack labelling. For each meal, three food options with different nutritional compositions were given to the participants. The contents of total energy and fat, saturated fat, sugar and salt of the chosen options were calculated.

Results: There were no significant differences in baseline sociodemographic and anthropometric characteristics between participants regardless of the experimental condition in which they started. There were no carry-over effects between the experimental sequences. It was observed that when participants used the multiple-traffic-light GDA system they chose significantly less total energy (mean $-123 \cdot 1$ (sD 211.0) kJ (-29.4 (sD 50.4) kcal), $P<0 \cdot 001)$, sugar $(-4 \cdot 5$ (sD 4.6) g, $P<0.001)$, fat $(-2 \cdot 1$ (SD 4.5$) \mathrm{g}, P=0.006$ ), saturated fat $(-1 \cdot 0$ (SD 1.9$) \mathrm{g}$, $P=0.002)$ and salt $(-0.4(\mathrm{SD} 0.5) \mathrm{g}, P<0.001)$ than when they used the monochrome GDA system.

Conclusions: Compared with the monochrome GDA front-of-pack nutritional label, the multiple-traffic-light system helped adolescents to differentiate between healthier and less healthy food, theoretically making it possible for them to choose a diet closer to dietary recommendations.
\end{abstract}

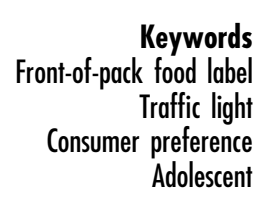

In many parts of the world, food industries and distributors, consumers and governments are re-examining the provision of nutritional information on food packages $^{(1-7)}$. The nutrition label is an important vehicle through which food manufacturers can communicate essential information about the nutritional value and composition of their product. Consumers are interested in the nutritional quality of food products and need transparent nutritional information about their purchases ${ }^{(8)}$. It is important that the nutritional information provided be appropriate and understandable to the consumer and that it has a positive impact on food-choice behaviours.
Potentially, food labelling is a valuable tool to help consumers make informed decisions about their diet in order to improve health and prevent chronic diseases.

In recent years, mandatory nutritional labelling requirements have been established in several countries including the $\mathrm{USA}^{(4)}$, the $\mathrm{EU}^{(3)}$, Australia and New Zealand ${ }^{(5)}$. In the EU countries, recent legislation ${ }^{(2)}$ has aimed to help consumers make healthy choices by requiring that nutritional information be listed on packaged food in the form of a nutritional information panel. When used by qualified health professionals this information is highly informative, but consumers find it difficult to understand ${ }^{(9)}$. 
In addition to the customary back-of-pack table labels, recently several food manufacturers and distributors have begun using signposts on the front of packages to help consumers interpret nutritional information ${ }^{(10)}$. Consumers, in general, like the idea of simplified frontof-pack information but have different preferences for the various formats created $^{(11)}$ : Guideline Daily Amounts (GDA), colour-coded indicators of nutrient levels (traffic lights) or healthy logos such as Sweden's Green Keyhole $^{(12)}$ or the Australian Heart Foundation 'Pick the Tick' sign ${ }^{(13)}$. The GDA show the total amount of energy and nutrients as a percentage of what a typical healthy adult should be eating daily on the basis of a $8374 \mathrm{~kJ}$ ( $2000 \mathrm{kcal})$ diet. The traffic-light labels (simple or multiple) give information about the level (i.e. high, medium or low) of individual nutrients in the product using the colour coding of red, yellow or green. The colour-coded GDA combines the two previous label systems. Studies carried out by the UK Food Standards Agency have shown that the colour-coded labels, like the multiple-traffic-light and coloured GDA formats, are the most widely accepted and best understood by consumers ${ }^{(14,15)}$.

However, differences in consumer preferences for the various formats may be related to age and to conflicting appreciations of ease of use, being fully informed and not feeling pressured into behaving in a particular way. Most consumers understand the most common signposting formats $^{(16)}$. However, little is known about how labelling information can affect consumers' dietary patterns ${ }^{(9,17)}$.

Nutrition labels are perceived as highly credible sources of information and many consumers use nutrition labels to guide their selection of food products. However, the extent to which labels are used varies considerably across subgroups: use is lowest among adolescents, older adults and individuals with low income or education or who are obese, and highest among those individuals with a healthier lifestyle and $\operatorname{diet}^{(1)}$.

In general the behaviour associated with the use of food labels has been studied in adults, but little is known about adolescents. To study how adolescents behave in response to nutrition labels is of great importance, because the habits formed in childhood are generally consolidated in adolescence and will remain in adulthood $^{(18)}$. In addition, adolescents are often very sensitive about their diet and body image $\mathrm{e}^{(19,20)}$ and at the same time very vulnerable to social food marketing ${ }^{(21)}$. There is strong evidence that marketing can influence young people's preferences and purchases ${ }^{(22)}$. Adolescents are also highly sensitive to external influences. For example, several studies have found that higher television watching is associated with an unhealthy dietary pattern ${ }^{(23)}$.

Unfortunately, very few studies have examined the impact of nutrition labels on adolescents ${ }^{(24-27)}$. In general, these studies have indicated that adolescents make little use of nutritional labels. In a study conducted in an urban primary-care clinic in the USA, only $22 \%$ of adolescents reported that they 'always' read the nutrition labels, $57 \%$ 'sometimes' and only $22 \%$ reported 'never' reading them $^{(24)}$. In addition, contradictory results have been reported in the literature about food-label use after interventions designed to promote their use. For example, evaluations of a 2006 US web-based nutrition intervention in adolescents found no improvement in food-label use as a result of the intervention ${ }^{(26)}$; in contrast, a similar 2008 study found that web-based interventions increased adolescents' use of labels ${ }^{(27)}$.

Therefore, the aim of the present study was to compare how adolescents in experimental food-choice conditions used two models of simplified front-of-pack GDA nutritional information which differed only in their use, or not, of colour indicators of the nutrient level. Particular attention was paid to two aspects: (i) adolescent friendliness and acceptance and (ii) the ability of adolescents to choose a diet that closely follows nutritional recommendations.

\section{Materials and methods}

\section{Study populations}

Eighty-one healthy participants of both sexes aged between 14 and 16 years were recruited from a state secondary school in Castellon (Spain). Exclusion criteria for the present study were: (i) refusal or inability (lack of capacity) to give informed consent; (ii) presence of an eating disorder; (iii) intentional or unintentional weight loss of more than $5 \%$ over the previous 3 months; (iv) diet modification as a result of metabolic or endocrine diseases; (v) presence of a major psychiatric condition; (vi) taking medication for chronic conditions; and (vii) professional family relationship with the food industry. The Scientific Committee of the Institut d'Investigació Sanitària Pere Virgili, and the board of directors of the school, approved the study protocol.

\section{Study design}

A randomized cross-over study was designed to compare two simplified front-of-pack nutrition labels. Participants were randomly assigned to two experimental sequences of exposure to nutritional labels: (i) multiple-traffic-light GDA (MTL-GDA) system - monochrome GDA (M-GDA) system; and (ii) M-GDA system - MTL-GDA system. A washout period of 1-3 weeks between the first and second experimental conditions was used to test possible interactions between experimental condition and sequence order (carry-over effect).

\section{Procedure}

Before exposing the participants to the experimental conditions, the researchers explained each of the frontof-pack nutritional labelling systems used in the study to the volunteers. Neither of the systems was encouraged or discouraged. It was explained that the purpose of the 
Table 1 Energy and nutrient composition of the proposed menus in comparison to the GDA for women

\begin{tabular}{|c|c|c|c|c|c|}
\hline \multirow[b]{2}{*}{ Nutrient } & \multirow[b]{2}{*}{ GDA for women } & \multicolumn{4}{|c|}{ Menus proposed } \\
\hline & & Unhealthy* & $\% G D A$ & Healthiert & $\% G D A$ \\
\hline \multicolumn{6}{|l|}{ Energy } \\
\hline $\mathrm{kJ}$ & 8374 & 8792 & 105 & 5949 & 71 \\
\hline kcal & 2000 & 2100 & 105 & 1421 & 71 \\
\hline Fat $(\mathrm{g})$ & 70 & 96.9 & 138 & $47 \cdot 0$ & 67 \\
\hline Saturated fat $(\mathrm{g})$ & 20 & $33 \cdot 8$ & 169 & $16 \cdot 9$ & 84 \\
\hline Sugar $(g)$ & 90 & $117 \cdot 3$ & 130 & $64 \cdot 8$ & 72 \\
\hline Salt (g) & 6 & $11 \cdot 5$ & 191 & $5 \cdot 0$ & 84 \\
\hline
\end{tabular}

study was to investigate whether food labels help to identify the 'healthier variant' of different foods.

Immediately after randomization, a self-administered questionnaire on demographic and personal data, body weight and height was completed by each participant. The socio-economic level was evaluated by the modified Hollingshead Index of Social Position ${ }^{(28)}$, which divides social level into five different classes: upper, upper middle, middle, lower middle and lower. We recoded them into tertiles: low socio-economic level (lower), middle socio-economic level (lower middle and middle) and high socio-economic level (upper middle and upper).

Once the questionnaire had been completed, participants had to choose options from an experimental menu (using a paper notebook with tabs) for breakfast, lunch, snack and dinner for $5 \mathrm{~d}$ according to the experimental simplified front-of-pack nutritional condition assigned (see the example of a lunch in the online supplementary material). Participants were exposed for a maximum of $15 \mathrm{~min}$ to each experimental condition. For each meal, three food options with different nutritional compositions were given to each participant. In both experimental conditions the energy and nutrient composition of the daily menus were the same and close to the reference dietary intakes (Table 1). The mean daily differences in the amount of energy and nutrients offered in the healthier option $v$. the less healthy option were $2843 \mathrm{~kJ}$ ( $679 \mathrm{kcal}$ ), $50 \cdot 0 \mathrm{~g}$ of total fat, $16 \cdot 0 \mathrm{~g}$ of saturated fat, $52.5 \mathrm{~g}$ of sugar and $6.5 \mathrm{~g}$ of salt.

After completing the experiment, the adolescents' response to the labels was assessed with two questions: (i) 'Which of the two simplified front-of-pack nutrition labels is the most helpful for choosing a healthy food option?' and (ii) 'Which of the methods is easiest to use when choosing a healthy diet?' They were also asked about energy-restricted diet antecedents and about their perception of their own body weight/shape (normal weight, overweight or obesity).

\section{Monochrome and multiple-traffic-light Guideline Daily Amounts systems}

The online supplementary material shows the options for a lunch using the M-GDA (Panel A) and the MTL-GDA (Panel B) front-of-pack label systems.
Table 2 Cut-offs used for traffic-light labels for a usual food serving

\begin{tabular}{lrccc}
\hline Nutrient & GDA & $\begin{array}{c}\text { Low (green) } \\
<7 \cdot 5 \% G D A\end{array}$ & $\begin{array}{c}\text { Moderate } \\
\text { (yellow) }\end{array}$ & $\begin{array}{c}\text { High (orange) } \\
>20 \% G D A\end{array}$ \\
\hline Energy & & & & \\
$\quad$ kJ & 8374 & $\leq 628$ & $628-1675$ & $\geq 1675$ \\
$\quad$ kcal & 2000 & 150 & $150-400$ & 400 \\
Fat (g) & 70 & $\leq 5 \cdot 25$ & $5 \cdot 25-14$ & $\geq 14$ \\
Saturated fat (g) & 20 & $\leq 1 \cdot 5$ & $1.5-4$ & $\geq 4$ \\
Sugar (g) & 60 & $\leq 4 \cdot 5$ & $4 \cdot 5-12$ & $\geq 12$ \\
Salt (g) & 6 & $\leq 0.45$ & $0.45-1.2$ & $\geq 1.2$ \\
\hline
\end{tabular}

GDA, Guideline Daily Amounts.

The MTL-GDA system for our study used green, yellow and orange to represent a low, moderate and high content, respectively, of selected nutrients on the front of food-product packs. Orange was used instead of red to avoid the stigmatization of any food as forbidden. The cutoff to establish the traffic-light colours was based on criteria from the UK Food Standards Agency ${ }^{(29)}$, but expressed in terms of a usual serving not $100 \mathrm{~g}$ of product.

The GDA used for labelling were based on the average requirements of an adult $\operatorname{woman}^{(30)}$ : energy intake, $8374 \mathrm{~kJ} / \mathrm{d}(2000 \mathrm{kcal} / \mathrm{d})$; total non-milk extrinsic sugars, $60 \mathrm{~g} / \mathrm{d}$; total fat, $70 \mathrm{~g} / \mathrm{d}$; saturated fat, $20 \mathrm{~g} / \mathrm{d}$; and salt, $6 \mathrm{~g} / \mathrm{d}$. A serving was coded as orange when it contained more than $20 \%$ of the GDA for energy or nutrient intake; green when it contained less than $7.5 \%$ of the GDA; and yellow when the value was between these two categories. We chose the GDA values for women because they simplify front-of-pack labels and are endorsed by experts as a good benchmark for the general population because they help consumers to avoid overconsumption. Table 2 shows the traffic-light cut-off used per serving.

When servings exceeded $250 \mathrm{~g}$, the following cut-offs were used to assign them to the orange category: total fat, $\geq 21 \mathrm{~g} /$ serving; saturated fat, $\geq 6 \mathrm{~g} / \mathrm{serving}$; total sugars, $\geq 18 \mathrm{~g} /$ serving; and salt, $\geq 24 \mathrm{~g} /$ serving.

\section{Outcome variables}

The average contents of energy, total and saturated fat, sugar and salt were calculated using the 5-d options 
Table 3 General characteristics of the study participants depending on the order in which they were subject to the two experimental conditions: healthy adolescents ( $n$ 81) aged $14-16$ years, Castellon, Spain

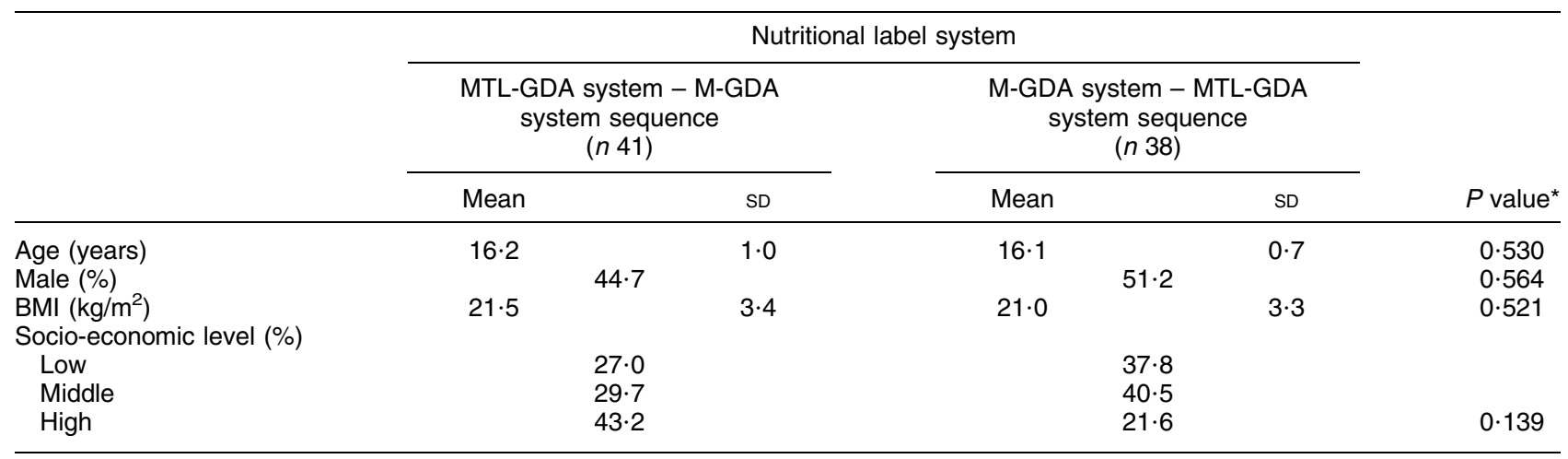

MTL-GDA, multiple-traffic-light Guideline Daily Amounts; M-GDA, monochrome Guideline Daily Amounts.

${ }^{\star} P$ value using the unpaired Student $t$ test or the $\chi^{2}$ test.

chosen by each participant in both experimental conditions and the commercial nutritional information.

\section{Statistical analysis}

Continuous variables are presented as the mean and standard deviation for normally distributed data, as the median and interquartile range for non-normally distributed data, and as percentages for categorical variables.

Comparisons between categorical variables were tested by the $\chi^{2}$ test. For comparisons of mean values of continuous variables, the Student $t$ test or the Mann-Whitney test for unpaired samples was used.

The possible interaction between the experimental condition sequences (carry-over effect) was tested as suggested by Senn ${ }^{(31)}$. A Student $t$ test was used to compare the mean values of the sum of the two experimental conditions between the group that began with the MTL-GDA system and the group that began with the M-GDA system. To assess the effect of the label system used, the Student $t$ test was used to compare the same two groups in terms of carry-over effect. On this occasion, however, the value obtained was the first period subtracted from the second period ${ }^{(31)}$.

Two-tailed $P$ values of $<0.05$ were considered significant. Statistical analyses were made with the statistical software package SPSS version $19 \cdot 0$.

\section{Results}

\section{Study sample}

Ninety-four students were contacted as eligible participants for the study. Of these, thirteen were not included because they met the exclusion criteria, and two were lost in follow-up because they did not complete the second experimental condition. Thus, a total of seventy-nine individuals were considered in the statistical analysis.

The general characteristics of the two populations that were subjected to both experimental conditions in different orders are described in Table 3. There were no significant differences in sex, age, BMI or socio-economic level between the participants who started the study using the MTL-GDA or the M-GDA front-of-pack foodlabel experimental condition.

\section{History of restricted diet and body weight perception}

A total of $10 \cdot 1 \%$ of the participants had been on an energy-restricted diet in the past. Most of the participants (78.5\%) perceived their body shape and weight to be normal, and $15 \cdot 2 \%$ regarded themselves as being overweight. A total of $97 \cdot 5 \%$ of participants considered that dietary pattern could have an important influence on their health, but this perception was not different between girls and boys $\left(\chi_{1}^{2}=0.003 ; P=0.957\right)$.

Almost ninety per cent $(89.9 \%)$ of the study participants preferred the MTL-GDA nutritional label system because it was more friendly and understandable, whereas only $2 \cdot 5 \%$ preferred the M-GDA label system.

\section{Intervention results}

No carry-over effect was observed between the experimental sequences on energy intake $(t=1 \cdot 2, \mathrm{df}=77$, $P=0 \cdot 231)$, sugar $(t=-0 \cdot 1, \mathrm{df}=73 \cdot 5, P=0 \cdot 898)$, total fat $(t=1 \cdot 8, \mathrm{df}=76 \cdot 4, P=0 \cdot 072)$, saturated fat $(t=1 \cdot 6, \mathrm{df}=77$, $P=0 \cdot 110)$ or salt $(t=0 \cdot 9, \mathrm{df}=75 \cdot 3, P=0 \cdot 371)$.

Table 4 shows that participants using the MTL-GDA system chose significantly less energy $(-123 \cdot 1$ (SD 211 0$)$ $\mathrm{kJ}(-29 \cdot 4(\mathrm{sD} 50 \cdot 4) \mathrm{kcal}), t=-3 \cdot 6, \mathrm{df}=66 \cdot 4, P=0 \cdot 001)$ and fewer nutrients $(-4 \cdot 5(\mathrm{sD} 4 \cdot 6) \mathrm{g}, t=-6 \cdot 2$, df $=65 \cdot 1$, $P<0.001$ for sugar; $-2 \cdot 1 \quad$ (SD 4.5$) \mathrm{g}, \quad t=-2 \cdot 8$, $\mathrm{df}=60 \cdot 3, P=0 \cdot 006$ for total fat; $-1 \cdot 0(\mathrm{sD} 1 \cdot 9) \mathrm{g}, t=-3 \cdot 2$, $\mathrm{df}=60 \cdot 8, P=0 \cdot 002$ for saturated fat; and $-0 \cdot 4(\mathrm{sD} 0 \cdot 5) \mathrm{g}$, $t=-5 \cdot 4, \mathrm{df}=75 \cdot 6, P<0 \cdot 001$ for salt) than those using the M-GDA labels. It should be noted that the differences between the most and the least healthy options represent $4 \cdot 3 \%, 8 \cdot 6 \%, 4 \cdot 1 \%, 5 \cdot 8 \%$ and $6 \cdot 0 \%$ less energy, sugar, total fat, saturated fat and salt, respectively, when the 
Table 4 Energy and nutrient differences chosen by participants using the MTL-GDA or the M-GDA system: healthy adolescents ( $n$ 81) aged $14-16$ years, Castellon, Spain

\begin{tabular}{|c|c|c|c|c|}
\hline & \multicolumn{2}{|c|}{ Difference between M-GDA and MTL-GDA systems } & \multirow{2}{*}{$\begin{array}{l}\text { Difference between the healthiest and least } \\
\text { healthy options expressed as a percentage }\end{array}$} & \multirow[b]{2}{*}{$P$ value* } \\
\hline & Mean & SD & & \\
\hline Energy (kJ) & $-123 \cdot 1$ & $211 \cdot 0$ & $-4 \cdot 3$ & $0 \cdot 0001$ \\
\hline Sugar $(\mathrm{g})$ & -4.5 & $4 \cdot 6$ & $-8 \cdot 6$ & $<0.001$ \\
\hline Total fat $(\mathrm{g})$ & $-2 \cdot 1$ & 4.5 & $-4 \cdot 1$ & 0.006 \\
\hline Saturated fat $(g)$ & $-1 \cdot 0$ & 1.9 & $-5 \cdot 8$ & 0.002 \\
\hline Salt $(g)$ & -0.4 & 0.5 & $-6 \cdot 0$ & $<0.001$ \\
\hline
\end{tabular}

MTL-GDA, multiple-traffic-light Guideline Daily Amounts; M-GDA, monochrome Guideline Daily Amounts.

${ }^{*} P$ value using the Student $t$ test for a single sample.

MTL-GDA nutritional label system was used. The differences between the energy and nutrients chosen using both label systems are independent of the participant's socio-economic level and gender (data not shown).

\section{Discussion}

Recently, the Food and Nutrition Board of the Institute of Medicine of the National Academies has recommended moving away from front-of-package systems that mostly provide nutritional information on foods or beverages but do not give clear guidance about their healthfulness, towards a system that encourages healthier choices through simplicity, visual clarity and the ability to convey meaning without written information ${ }^{(32)}$. So, it is of great interest to investigate how different types of front-of-pack nutritional labelling formats affect food choice, purchase and consumption.

To our knowledge, few studies have evaluated the effect that different types of front-of-pack nutritional labels have on consumer food choices, and none of the studies that have been carried out have focused on adolescents. We focused on adolescents because they are often very sensitive about their diet and body image and at the same time very vulnerable to marketing techniques used to influence consumer choices. Furthermore, habits formed early in life are generally consolidated in adolescence and will remain during adulthood.

The present cross-over study indicated that the population studied chose a significantly different diet when they used a colour-based traffic-light GDA front-of-pack label compared with the same monochrome model. The colours used in the multiple-traffic-light system helped participants to identify healthy and less healthy products, and to differentiate between them, thus making it possible for them to choose a diet that followed dietary recommendations more closely.

The most probable and robust explanation for our findings is that the MTL-GDA nutritional label is more immediately comprehensible to consumers and easier to interpret. Therefore, it allows them to choose foods with fewer kilojoules, sugar, fats and salt. In fact, almost ninety per cent of the adolescents preferred the multipletraffic-light system because it was more friendly and understandable, whereas less than $3 \%$ preferred the monochrome system.

Several studies have evaluated the effect of different types of front-of-pack nutritional labels on the ability of consumers to differentiate healthier food products ${ }^{(15,33,34)}$. Kelly and co-workers ${ }^{(33)}$, for example, used a sample of 790 adults from New South Wales, Australia to compare four front-of-pack nutritional labelling systems: a monochrome percentage Daily Intake (\%DI) system; a colourcoded \%DI; and two variations of traffic-light systems which used colour coding to indicate nutrient levels. On the other hand, the consumers using traffic-light labels were five and three times more likely to correctly identify the healthier food products than the consumers using the monochrome or the colour-coded \%DI systems, respectively, after adjusting for sex, education level and household income ${ }^{(27)}$. No differences were observed between the monochrome and the colour-coded \%DI systems, probably because they represent eight nutrients and not just the four or five nutrients represented by the traffic-light labels. In addition, the results of the study by Kelly et $a l .{ }^{(33)}$ suggest that the traffic-light system more effectively allows consumers of a lower socio-economic status - that is to say, those who are most at risk of obesity - to identify healthy products ${ }^{(34)}$. This is not the case in the present study, because socio-economic level was not observed to lead to any differences (data not shown).

Various studies have demonstrated that if a limited number of nutrients are represented by means of traffic lights, consumer acceptability increases ${ }^{(14,15,33)}$. Trafficlight labelling formats led to high levels of understanding and acceptance across ethnic and income groups in a survey of 1525 shoppers in New Zealand ${ }^{(34)}$. A randomized experimental study conducted on 420 adults from Hamburg, Germany demonstrated that multiple-trafficlight labels helped consumers differentiate between healthy foods and less healthy foods, particularly in relation to body weight. Nevertheless, such changes in perceived healthiness were unlikely to influence food choice or consumption $^{(15)}$. In a study conducted with ninety-two adults, Jones and Richardson also found that 
the traffic-light system, unlike the standard nutritional label, helped to guide the attention of the consumer to the important nutrients and improved the accuracy of the health ratings of nutrition labels ${ }^{(35)}$.

Although front-of pack nutrition labelling seems to help consumers make healthier food choices, lack of attention to these labels can limit their effectiveness. Recently, it has been shown that although consumers evaluate nutritional tables positively, these tables receive little attention and do not prompt healthy options ${ }^{(36)}$. The previous study demonstrated that traffic-light labels and logos enhance healthy product choice, even when consumers are subject to the pressure of time. So, it is important to know the conditions in which subjects are surveyed if the results are to be correctly interpreted. In this regard, our survey lasted for only $35 \mathrm{~min}$, which reflects real purchase conditions. This time is probably too short to correctly identify the healthiest options for $5 \mathrm{~d}$.

Our study has strengths and weaknesses. Among its strengths we should highlight the randomized cross-over design and that the baseline variables were controlled between both experimental groups. In addition, to ensure that our findings could be attributed to the differences in the front-of-pack labelling, the food packages in our study did not show other relevant nutritional information such as lists of ingredients, nutritional claims or brand names, which may have had an influence on survey responses.

Our study also has several limitations. First, our sample was recruited from only one school. Therefore, our findings cannot be extrapolated to the general population. Another important limitation is that we assessed only potential food choice and not real shopping or consumption. It was a non-real food-choice experimental situation. Therefore, the results may not reflect exactly what would happen in a real context. In this regard, very few studies have tested consumption or purchases in real conditions. Temple et al. found that traffic-light labels can increase consumption of more healthy foods and decrease consumption of the less healthy ones in lunches consumed in laboratory conditions ${ }^{(37)}$. Although not all research supports the notion that traffic-light nutritional labelling is likely to have an effect on behaviour ${ }^{(10)}$, some authors argue that traffic-light labelling may impact consumer purchasing patterns only in the longer term or if labelling covers a wide range of products ${ }^{(38)}$.

Bearing these limitations in mind, we conclude that the traffic-light GDA front-of-pack label may help adolescents to more readily identify products with lower amounts of energy, sugars, total fats and salt than a monochrome model. Nutritional labels are used relatively little among adolescents but, even so, our adolescents were able to construct a diet with fewer nutrients and less energy, which in a different situation and from the point of view of the prevalence of childhood/youth obesity and adult chronic disease would be beneficial. However, future research into labelling should determine the impact that nutritional labels can have on consumer behaviour and be extended to other population groups.

\section{Acknowledgements}

Sources of funding: This study was funded, in part, by the Eroski Foundation (Spain), the Spanish Ministry of Health (Instituto de Salud Carlos III), Thematic Network RTIC RD06/0045/0009 and FEDER (Fondo Europeo de Desarrollo Regional). CIBERobn is an initiative of the Instituto de Salud Carlos III, Spain. None of the funding sources played a role in the design, collection, analysis or interpretation of the data or in the decision to submit the manuscript for publication. Conflict of interest: N.B and J.S.-S. have received grants from the Eroski Foundation. None of the other authors have conflicts of interest affecting the conduct or reporting of the work submitted. Authors' contributions: N.B. contributed to the design of the study, acquisition and analysis of the data, interpretation of the results and drafting of the manuscript. P.V., L.L. and A.B. contributed to the acquisition and analysis of the data and the interpretation of results. J.B. contributed to the conception of the study, interpretation of the data and drafting of the manuscript. J.S.-S. contributed to the design and coordination of the study, interpretation of the results and drafting of the manuscript with intellectual and scientific input. All authors revised and approved the submitted manuscript. Acknowledgements: The authors thank participants and Fundación Eroski for their support in carrying out this study.

\section{Supplementary material}

To view supplementary material for this article, please visit http://dx.doi.org/10.1017/S1368980013001274

\section{References}

1. Campos S, Doxey J \& Hammond D (2011) Nutrition labels on pre-packaged foods: a systematic review. Public Health Nutr 14, 1496-1506.

2. Commission of the European Communities (2011) Regulation (EU) No. 1169/2011 of the European Parliament and of the Council. Official Journal of the European Union 22.11.2011, L308/18-L304/63. http://eur-lex.europa.eu/Lex UriServ/LexUriServ.do?uri=OJ:L:2011:304:0018:0063:EN:PDF (accessed December 2012).

3. Commission of the European Communities (2008) Proposal for a regulation of the European Parliament and of the Council on the provision of food information to consumers. $\operatorname{COM}(2008) 40$ final. http://ec.europa.eu/food/ food/labellingnutrition/foodlabelling/publications/proposal_ regulation_ep_council.pdf (accessed October 2011).

4. US Food and Drug Administration (2011) Food labeling and nutrition. http://www.fda.gov/Food/GuidanceCompliance RegulatoryInformation/GuidanceDocuments/FoodLabeling Nutrition/default.htm (accessed December 2012).

5. Food Standards Australia New Zealand (2012) Food labelling. http://www.foodstandards.gov.au/consumerinformation/ labellingoffood/ (accessed December 2012). 
6. Health Canada (2010) Nutrition Labelling. http://www.hc-sc. gc.ca/fn-an/label-etiquet/nutrition/index-eng.php (accessed December 2012).

7. Mhurchu CN \& Gorton D (2007) Nutrition labels and claims in New Zealand and Australia: a review of use and understanding. Aust N Z J Public Health 31, 105-112.

8. Wills JM, Schmidt DB, Pillo-Blocka F et al. (2009) Exploring global consumer attitudes toward nutrition information on food labels. Nutr Rev 67, Suppl. 1, S102-S106.

9. Cowburn G \& Stockley L (2005) Consumer understanding and use of nutrition labelling: a systematic review. Public Health Nutr 8, 21-28.

10. Feunekes GI, Gortemaker IA, Willems AA et al. (2008) Front-of-pack nutrition labelling: testing effectiveness of different nutrition labelling formats front-of-pack in four European countries. Appetite 50, 57-70.

11. Möser A, Hoefkens C, Camp J et al. (2010) Simplified nutrient labelling: consumers' perceptions in Germany and Belgium. J Consum Protect Food Saf 5, 169-180.

12. Larsson I, Lissner L \& Wilhelmsen L (1999) The 'green keyhole' revisited: nutritional knowledge may influence food selection. Eur J Clin Nutr 53, 776-780.

13. Eyles H, Gorton D \& Ni Mhurchu C (2010) Classification of 'healthier' and 'less healthy' supermarket foods by two Australasian nutrient profiling models. $N Z$ Med J 123, 8-20.

14. Malam S, Clegg S, Kirwan S et al. (2012) Comprehension and use of UK nutrition signpost labelling schemes. http:// www.food.gov.uk/multimedia/pdfs/pmpreport.pdf (accessed February 2012).

15. Borgmeier I \& Westenhoefer J (2009) Impact of different food label formats on healthiness evaluation and food choice of consumers: a randomized-controlled study. BMC Public Health 9, 184.

16. Grunert KG, Fernandez-Celemin L, Wills JM et al. (2010) Use and understanding of nutrition information on food labels in six European countries. $Z$ Gesundh Wiss 18, 261-277.

17. Sacks G, Rayner M \& Swinburn B (2009) Impact of front-ofpack 'traffic-light' nutrition labelling on consumer food purchases in the UK. Health Promot Int 24, 344-352.

18. Dain E (2012) Feeding and adolescence. Rev Med Brux 33, 346-353.

19. Verri AP, Verticale MS, Vallero E et al. (1997) Television and eating disorders. Study of adolescent eating behavior. Minerva Pediatr 49, 235-243.

20. Friederich HC, Uher R, Brooks S et al. (2007) I'm not as slim as that girl: neural bases of body shape self-comparison to media images. Neuroimage 37, 674-681.

21. Bryant CA, Mayer AB, McDermott RJ et al. (2011) Social marketing: an underutilized tool for promoting adolescent health. Adolesc Med State Art Rev 22, 387-401.

22. Institute of Medicine (2006) Food Marketing to Children and Youth: Threat or Opportunity? http://www.iom.edu/ / media/Files/Report\%20Files/2005/Food-Marketing-to-Childrenand-Youth-Threat-or-Opportunity/KFMOverviewfinal2906.pdf (accessed December 2012).
23. Sisson SB, Shay CM, Broyles ST et al. (2012) Televisionviewing time and dietary quality among US children and adults. Am J Prev Med 43, 196-200.

24. Huang TT, Kaur H, McCarter KS et al. (2004) Reading nutrition labels and fat consumption in adolescents. $J$ Adolesc Health 35, 399-401.

25. McCullum C \& Achterberg CL (1997) Food shopping and label use behavior among high school-aged adolescents. Adolescence 32, 181-197.

26. Long JAD, Armstrong ML, Amos E et al. (2006) Pilot using world wide web to prevent diabetes in adolescents. Clin Nurs Res 15, 67-79.

27. Abood DA, Black DR \& Coster DC (2008) Evaluation of a school-based teen obesity prevention minimal intervention. J Nutr Educ Behav 40, 168-174.

28. Hollingshead A (1975) Four Factor Index of Social Position. New Haven, CT: Yale University Department of Sociology Press.

29. Food Standards Agency (2007) Front-of-Pack Traffic Light Signpost Labeling Technical Guidance. London: Food Standards Agency.

30. European Food Information Council (2007) Making sense of Guideline Daily Amounts. http://www.eufic.org/article/ en/nutrition/food-labelling-claims/artid/Making_sense_of_ Guideline_Daily_Amounts/ (accessed December 2012).

31. Senn S (2002) Cross-Over Trials in Clinical Research, 2nd ed. Chichester: John Wiley \& Sons, Ltd.

32. Food and Nutrition Board, Institute of Medicine of the National Academies (2011) Front-of-Package Nutrition Rating Systems and Symbols: Promoting Healthier Choices. Consensus Report. http://www.iom.edu/Reports/2011/ Front-of-Package-Nutrition-Rating-Systems-and-SymbolsPromoting-Healthier-Choices.aspx (accessed December 2011).

33. Kelly B, Hughes C, Chapman K et al. (2009) Consumer testing of the acceptability and effectiveness of front-ofpack food labelling systems for the Australian grocery market. Health Promot Int 24, 120-129.

34. Gorton D, Ni Mhurchu C, Chen MH et al. (2009) Nutrition labels: a survey of use, understanding and preferences among ethnically diverse shoppers in New Zealand. Public Health Nutr 12, 1359-1365.

35. Jones G \& Richardson M (2007) An objective examination of consumer perception of nutrition information based on healthiness ratings and eye movements. Public Health Nutr 10, 238-244.

36. van Herpen E \& Trijp HC (2011) Front-of-pack nutrition labels. Their effect on attention and choices when consumers have varying goals and time constraints. Appetite 57, 148-160.

37. Temple JL, Johnson KM, Archer K et al. (2011) Influence of simplified nutrition labeling and taxation on laboratory energy intake in adults. Appetite 57, 184-192.

38. Sacks G, Tikellis K, Millar L et al. (2011) Impact of 'trafficlight' nutrition information on online food purchases in Australia. Aust N Z J Public Health 35, 122-126. 